\title{
Ultrafast Real-time Dynamics of CO \\ Oxidation Over an Oxide Photocatalyst
}

Michael Wagstaffe, ${ }^{\dagger}$ Lukas Wenthaus, ${ }^{\dagger}+$ Adrian Dominguez-Castro, ${ }^{\top}$ Simon

Chung, $^{\dagger}$ Guilherme Dalla Lana Semione, ${ }^{\dagger}$ Steffen Palutke, ${ }^{\dagger}$ Giuseppe Mercurio, ${ }^{\S}$ Siarhei Dziarzhytski, ${ }^{\dagger}$ Harald Redlin, ${ }^{\dagger}$ Nicolai Klemke, ${ }^{\ddagger}$ Yudong Yang, ${ }^{\ddagger}$ Thomas $^{-}$

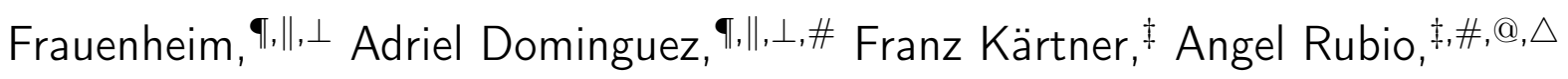
Wilfried Wurth, ${ }^{\dagger, \ddagger} \nabla$ Andreas Stierle, ${ }^{\dagger, \nabla}$ and Heshmat Noei ${ }^{*, \dagger}$

$\dagger$ Deutsches Elektronen-Synchrotron (DESY), Hamburg, Germany

$\ddagger$ Center for Free-Electron Laser Science, Hamburg, Germany

\Bremen Center for Computational Material Science (BCCMS), Bremen, Germany

$\S$ European XFEL GmbH, Schenefeld, Germany

\|Computational Science and Applied Research Institute (CSAR), Shenzhen, China

$\perp$ Beijing Computational Science Research Center (CSRC), Beijing, China

\#Nano-Bio Spectroscopy Group, Departamento de Fisica de Materiales, Universidad del

País Vasco, UPV/EHU- 20018 San Sebastián, Spain

@Max Planck Institute for the Structure and Dynamics of Matter, Hamburg, Germany

$\triangle$ Center for Computational Quantum Physics, Flatiron Institute, New York, USA

$\nabla$ Fachbereich Physik Universität Hamburg, Hamburg, Germany

E-mail: heshmat.noei@desy.de

\begin{abstract}
Femtosecond X-ray laser pulses synchronized with an optical laser were employed to
\end{abstract}


investigate the reaction dynamics of the photooxidation of $\mathrm{CO}$ on the anatase $\mathrm{TiO}_{2}(101)$ surface in real-time. Our time-resolved soft X-ray photoemission spectroscopy results provide evidence of ultrafast timescales and, coupled with theoretical calculations, clarify the mechanism of oxygen activation that is crucial to unraveling the underlying processes for a range of photocatalytic reactions relevant to air purification and selfcleaning surfaces. The reaction takes place between $1.2-2.8( \pm 0.2)$ ps after irradiation with an ultrashort laser pulse leading to the formation of $\mathrm{CO}_{2}$, prior to which no intermediate species were observed on a picosecond time scale. Our theoretical calculations predict that the presence of intragap unoccupied $\mathrm{O}_{2}$ levels leads to the formation of a charge-transfer complex. This allows the reaction to be initiated following laser illumination at a photon energy of $1.6 \mathrm{eV}(770 \mathrm{~nm})$, taking place via a proposed mechanism involving the direct transfer of electrons from $\mathrm{TiO}_{2}$ to physisorbed $\mathrm{O}_{2}$.

keywords: time-resolved photoelectron spectroscopy, ultrafast, photocatalysis, photochemistry, oxygen activation, $\mathrm{TiO}_{2}$, metal oxide, free electron laser 


\section{Introduction}

Oxide semiconductors constitute an important class of materials capable of photochemically driven processes that are responsible for the efficient degradation of volatile organic compounds and pollutants, including carbon monoxide and $\mathrm{NO}_{x} \cdot{ }^{1-6} \mathrm{TiO}_{2}$ is one of the most promising candidates for applications in photocatalysis ${ }^{2,3,5,7}$ due to its low costs, excellent structural and mechanical properties, chemical stability and high photocatalytic activity. ${ }^{5}$ Studies of photoinduced reactions on $\mathrm{TiO}_{2}$ are thus instrumental to the development of efficient technology responsible for air and water purification and for self-cleaning surfaces. ${ }^{2,3,6,7}$ In the traditional picture, photocatalytic reactions are initiated by photon absorption that causes the promotion of electrons from the valence band to the conduction band of the material. ${ }^{2}$ Following the generation of electrons and holes $(\mathrm{e} / \mathrm{h})$, the charges can become trapped at surface active sites where they can drive a series of redox reactions. ${ }^{2,3,7}$ The activation of oxygen on $\mathrm{TiO}_{2}$ is often the crucial first step of many oxidation processes, leading to the formation of highly reactive intermediate species including the superoxide radical anion $\left(\bullet^{\bullet} \mathrm{O}^{-}\right)$, hydroxide radicals $(\bullet \mathrm{OH})$, hydroperoxy radicals $(\bullet \mathrm{OOH})$ and $\mathrm{O}^{2-} \cdot{ }^{6,8}$ Further to this, the quenching of charge carriers by oxygen is reported to suppress charge carrier recombination. ${ }^{1}$ The oxygen activation process, and the transient nature of intermediate oxygen species, governs the entire photocatalytic cycle, but a deep understanding thereof has remained elusive.

The photochemical oxidation of carbon monoxide $(\mathrm{CO})$ to carbon dioxide $\left(\mathrm{CO}_{2}\right)$ catalysed by $\mathrm{TiO}_{2}$ is an important reaction for air purification. ${ }^{9-13} \mathrm{CO}$ is thought to be responsible for a large number of fatal air poisonings in many industrial countries ${ }^{14,15}$ and therefore its abatement is of paramount importance. Applying X-ray Photoelectron Spectroscopy (XPS), we recently monitored the slow kinetics (on minute time scales) of the photooxidation of $\mathrm{CO}$ on $\mathrm{TiO}_{2} \cdot{ }^{16}$ In agreement with Infrared reflection-absorption spectroscopy (IRRAS) studies, ${ }^{10,17}$ faster kinetics were observed for anatase when compared to rutile, but detailed information concerning the early-stage reaction dynamics, the role of oxygen and how this influences the 
photochemistry could not be discerned due to the limited time resolution. Previous studies have suggested that the absence of surface carbonates during the photoreaction indicates that the reaction proceeds without forming reaction intermediates, ${ }^{9,18}$ this however could be a consequence of the short lifetime of these species. Thus far, the majority of studies concerning the photoactivity of $\mathrm{TiO}_{2}$ were carried out with a time resolution longer than picoseconds meaning that information on the ultrafast dynamics, which govern the efficiency of reactions important to heterogeneous catalysis, was lost. ${ }^{19,20}$

With the advent of X-ray free-electron lasers (FELs), the ability to probe the reaction dynamics of fundamental processes on their natural timescale took a significant step forward, allowing one to monitor charge carrier dynamics with an unprecedented temporal resolution. ${ }^{21-26}$ Here, we report on an ultrafast optical pump-X-ray probe photoemission study at the FEL FLASH, located at the Deutsches Elektronen-Synchrotron (DESY) in Hamburg. FLASH exploits superconducting RF accelerator technology to offer the high repetition rates necessary to observe and monitor rare events and subtle chemical changes with ultrafast time resolution. ${ }^{27,28}$ Capitalizing on this, we are able to resolve the reaction dynamics of the photoinduced oxidation of $\mathrm{CO}$ to $\mathrm{CO}_{2}$ on the anatase $\mathrm{TiO}_{2}(101)$ surface by directly monitoring the formation of reaction products on a picosecond time-scale with a time resolution of 194 fs. We demonstrate that $\mathrm{CO}_{2}$ formation takes place within the first $1.2( \pm 0.2)$ ps following laser illumination at a photon energy of $1.6 \mathrm{eV}(770 \mathrm{~nm})$. The work is combined with DFT and DFTB calculations, providing new insight concerning a reaction pathway initiated by the excitation of valence band electrons into intraband gap states appearing upon oxygen adsorption. This leads to the formation of an $\mathrm{O}_{2}-\mathrm{TiO}_{2}$ charge-transfer complex that can be activated at a photon energy below the band gap of $\mathrm{TiO}_{2}$. 


\section{Results and Discussion}

As a prerequisite for the assignment of the $\mathrm{O}$ 1s and $\mathrm{C}$ 1s core level signals relevant to $\mathrm{CO}$ and $\mathrm{CO}_{2}$ adsorption, we performed lab based XPS $(\mathrm{h} \nu=1486.6 \mathrm{eV})$ experiments. ${ }^{16}$ They demonstrated that $\mathrm{CO}$ and $\mathrm{CO}_{2}$ adsorb on the $\mathrm{TiO}_{2}$ surface with two clearly distinct peaks located at binding energies of $290.1 \mathrm{eV}$ and $291.7 \mathrm{eV}$ in the $\mathrm{C} 1 \mathrm{~s}$ core level $(\Delta \mathrm{E}=$ $1.6 \mathrm{eV})$ and $536.6 \mathrm{eV}$ and $535.5 \mathrm{eV}(\Delta \mathrm{E}=1.1 \mathrm{eV})$ in the $\mathrm{O}$ 1s core level, respectively, see supplementary information (SI) Figure S1. Additionally, we demonstrated that the slow reaction kinetics can be followed using XPS during exposure of the surface to $\mathrm{O}_{2}$ and UV light, see Figure S2. Conversely, simultaneous exposure of the CO covered surface to oxygen and X-rays $(1486.6 \mathrm{eV})$ for 30 minutes in the absence of UV light showed no changes, see Figure S3, reinforcing the fact that simultaneous exposure of the surface to UV light and oxygen is necessary. ${ }^{10,16}$ The system was then investigated using ultrafast optical pump-X-ray probe photoemission spectroscopy at the second branch of the plane grating monochromator beamline (PG2) $)^{29,30}$ of FLASH. ${ }^{27,28}$ FLASH provided a pulse pattern of bursts with $10 \mathrm{~Hz}$ repetition rate, consisting of 400 single X-ray pulses with a $1 \mathrm{MHz}$ repetition rate. After monochromator broadening, each FEL pulse had a FWHM temporal width of appropriately 150 fs. An optical laser $\left(\mathrm{h} \nu_{\text {opt }}=1.6 \mathrm{eV}\right)$ was synchronized with the FEL $\left(\mathrm{h} \nu_{F E L}=647.9\right.$ $\mathrm{eV})$ to pump the photocatalytic reaction with the relative temporal offset between the two pulses realized by a delay stage. For more details refer to the experimental section. The temporal overlap between the FEL and optical laser pulses was established using the process of laser-assisted photoemission via creation of the sidebands in the time-dependent Ti $2 \mathrm{p}$ and $\mathrm{O}$ 1s photoelectron signals, as described in ${ }^{31}$ and discussed in more detail in the SI, Figures S4 and S5. The temporal resolution of the experiment was determined to be $194 \pm$ 26 fs. After sample preparation and cooling to $60 \mathrm{~K}$, the surface was immediately exposed to a mixed atmosphere of $\mathrm{CO}\left(3 \times 10^{-8}\right.$ mbar $)$ and $\mathrm{O}_{2}\left(3 \times 10^{-8} \mathrm{mbar}\right)$. 


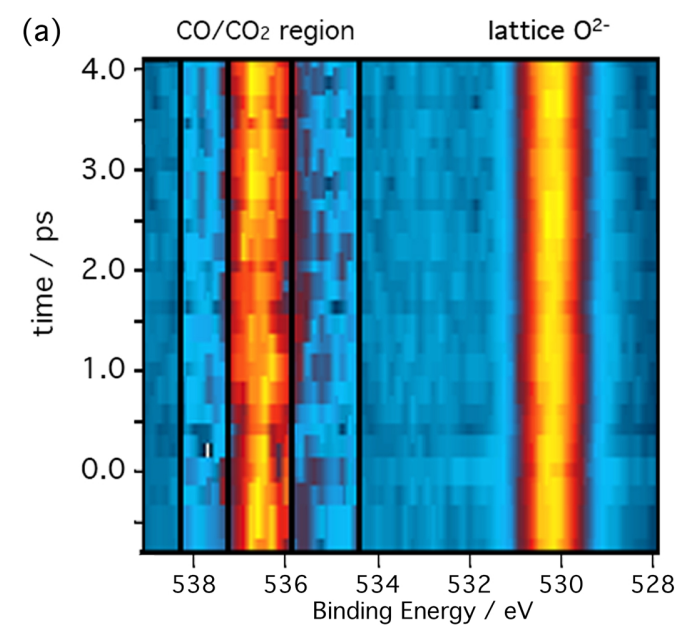

(b)

(c)
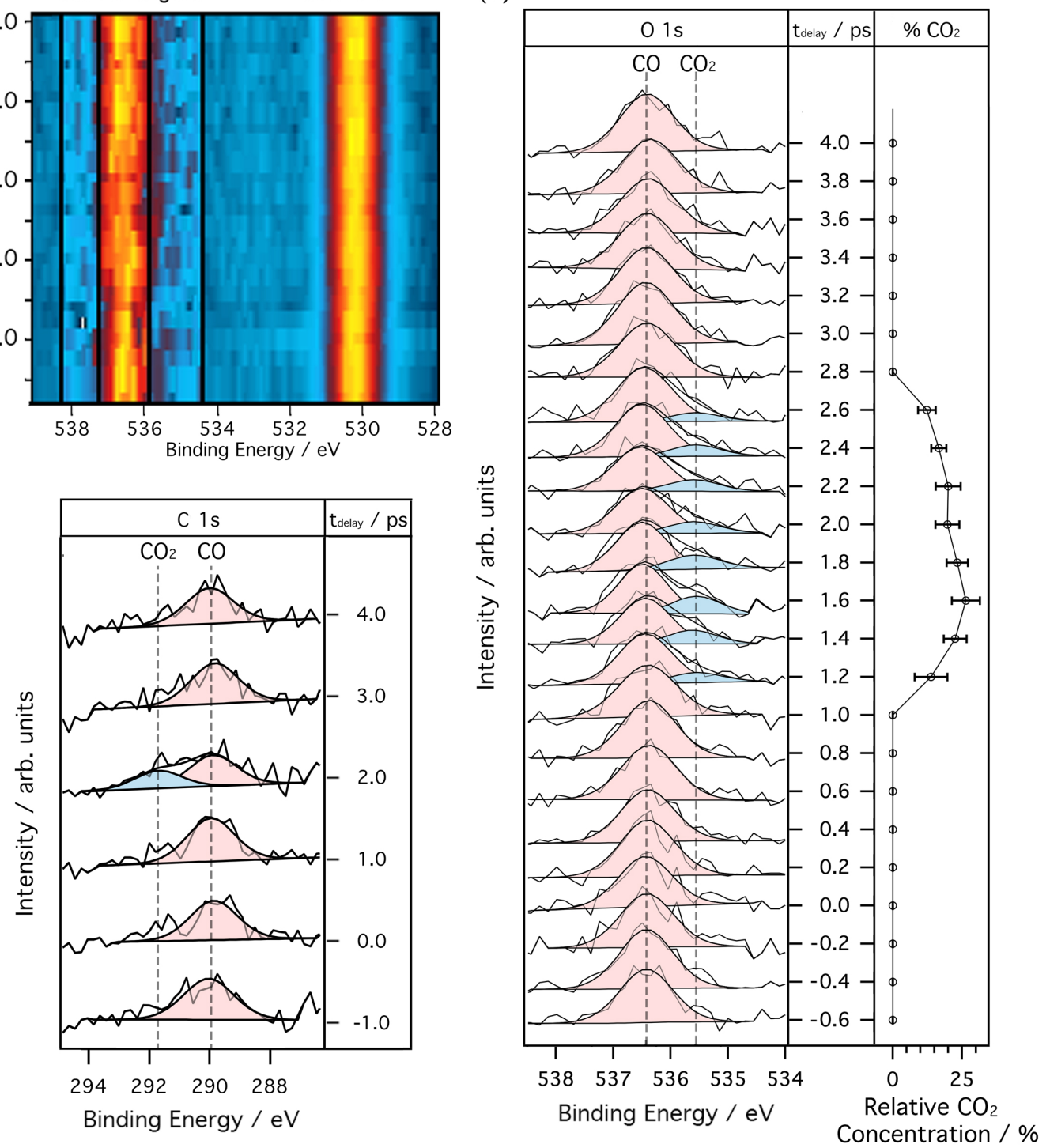

Figure 1: Time resolved XP spectra during the photooxidation of $\mathrm{CO}$ to $\mathrm{CO}_{2}$ on anatase $\mathrm{TiO}_{2}(101)\left(\mathrm{h} \nu_{F E L}=647.9 \mathrm{eV}, \mathrm{h} \nu_{o p t}=1.6 \mathrm{eV}, \mathrm{T}=60 \mathrm{~K}\right)$. (a) shows the time-resolved O1s XPS map recorded during the pump probe experiment between - $0.6 \mathrm{ps}$ and 4.0 ps delay time (temporal resolution of $194 \mathrm{fs}$ ). Highlighted is the $\mathrm{CO} / \mathrm{CO}_{2}$ region in which a low binding energy shoulder appears to grow between 1.0 and 3.0 ps. (b) O 1s core level spectra from (a) shown as a function of delay time averaged over $200 \mathrm{fs}$ windows. The relative concentration of $\mathrm{CO}_{2}$ is displayed to the right, with the corresponding errors determined from the fits to the spectra. (c) C 1s core level spectra, averaged over 1 ps windows between - 1.0 ps and +4.0 ps. The $\mathrm{O}$ 1s and $\mathrm{C}$ 1s core levels are deconvoluted as described in the experimental method and the components for $\mathrm{CO}$ and $\mathrm{CO}_{2}$ are displayed as solid lines centred on the dashed lines, with the enclosed area shaded in red and blue respectively. 
Figure 1(a) shows the time resolved core level O 1s XP map, recorded at FLASH, during the photooxidation of CO. Highlighted to accentuate the features in Figure 1(a) is the region containing the $\mathrm{O}$ 1s contributions of $\mathrm{CO}$ and $\mathrm{CO}_{2}$. A transfer of intensity to a lower binding energy shoulder of the $\mathrm{CO}$ peak, between $535 \mathrm{eV}$ and $536 \mathrm{eV}$, is apparent between 1 and 3 ps. In a further evaluation aimed to elucidate the origin of this transfer of intensity, the time-resolved O 1s XP map is displayed as a series of core level spectra and the temporal evolution of the $\mathrm{O}$ 1s core level signal between $-0.6 \mathrm{ps}$ and $4.0 \mathrm{ps}$ is shown in Figure 1(b). Between $-0.6 \mathrm{ps}$ and $1.0 \mathrm{ps}$ in the $\mathrm{O}$ 1s spectra no notable changes occur and only the signal of $\mathrm{CO}$ adsorbed on the surface is present at a binding energy of $536.5 \mathrm{eV}$. At $1.2 \pm 0.2 \mathrm{ps}$ a chemical state change indicative of the formation of $\mathrm{CO}_{2}$ is observed via the formation of a shoulder at binding energy $535.6 \mathrm{eV}$. The difference in binding energy shifts between $\mathrm{CO}$ and $\mathrm{CO}_{2}(\Delta \mathrm{E}=0.9 \mathrm{eV})$ when compared to the lab data ${ }^{16}$ shown in Figure $\mathrm{S} 1(\Delta \mathrm{E}=1.1$ $\mathrm{eV}$ ) is ascribed to coverage dependent shifts, which were reported to be up to $0.5 \mathrm{eV}$ for $\mathrm{CO}$ adsorption on anatase(101). ${ }^{32}$ Expected reaction intermediates, such as $\mathrm{CO}_{3}{ }^{2-},{ }^{33} \mathrm{COOH},{ }^{14}$ or other oxygen containing hydrocarbon species typically appear in the $\mathrm{O}$ 1s spectrum at lower binding energies in the range of $532.0 \mathrm{eV}-534.0 \mathrm{eV}$. ${ }^{5,34}$ Figure $\mathrm{S} 6$ displays the spectra over an extended binding energy range, highlighting that no additional species are observed within this range prior to the formation of $\mathrm{CO}_{2}$. Our data thus provides direct evidence that the photooxidation of $\mathrm{CO}$ to $\mathrm{CO}_{2}$ on anatase $\mathrm{TiO}_{2}(101)$ can proceed without forming carbon-containing intermediate species with a lifetime greater than 200 fs. Following this we observe that the relative amount of photogenerated $\mathrm{CO}_{2}$ on the surface increases over a period of $0.6 \mathrm{ps}(1.0-1.6 \mathrm{ps})$ before it decreases over a period of $1.2 \mathrm{ps}(1.6-2.8 \mathrm{ps})$. The implications of this are discussed later. To reinforce the claim of $\mathrm{CO}_{2}$ formation, see Figure $\mathrm{S} 7$ for the spectra averaged over a wider temporal window of $1 \mathrm{ps}$, consequently improving the signal to noise ratio but to the detriment of temporal resolution. After 2.8 ps the spectra once again comprises only of $\mathrm{CO}$, suggesting all photogenerated $\mathrm{CO}_{2}$ has desorbed. Over time, some $\mathrm{CO}_{2}$ will readsorb on the surface, as can be seen in the time averaged data shown 
in Figure S8. Nevertheless, the high back pressure of CO $\left(3 \times 10^{-8}\right.$ mbar $)$ means competitive readsorption of $\mathrm{CO}$ will likely dominate, allowing $\mathrm{CO}$ to readsorb before the next pump cycle $(+1 \mu \mathrm{s})$ and allowing the photoreaction to repeat itself in a cyclic manner. A movie of the photoreaction in the $\mathrm{O}$ 1s core level is shown in Movie S1. In excellent agreement with the O 1s spectra, the reaction dynamics are similarly observed in the $\mathrm{C}$ 1s core level. This is evidenced by a peak at $291.8 \mathrm{eV}$ corresponding to $\mathrm{CO}_{2}$ that is present only at $2.0 \pm 0.5$ ps, shown in Figure 1(c). As before, the slight difference in binding energy shifts between $\mathrm{CO}$ and $\mathrm{CO}_{2}(\Delta \mathrm{E}=1.8 \mathrm{eV})$ when compared to the lab data ${ }^{16}$ shown in Figure $\mathrm{S} 1(\Delta \mathrm{E}$ $=1.6 \mathrm{eV})$ is ascribed to coverage dependent shifts. ${ }^{32}$ The photoionisation cross-section at $647.9 \mathrm{eV}$ is approximately three times larger for oxygen compared to carbon ${ }^{35}$ and, due to the stoichiometry of the molecule, any relative changes observed in the $\mathrm{C}$ 1s core level are expected to be half as prominent as in the $\mathrm{O}$ 1s core level. Taking this into consideration, the $\mathrm{C}$ 1s data had to be averaged over a wider temporal window of 1 ps in order to achieve the necessary statistics for reliable peak fitting.

To rationalize our experimental observations, we performed first principles and tightbinding calculations for the adsorption of $\mathrm{O}_{2}$ and $\mathrm{CO}$ on the anatase $\mathrm{TiO}_{2}(101)$ surface. For DFT calculations describing the electronic and structural properties of the adsorption of $\mathrm{CO}$ and $\mathrm{O}_{2}$ in detail, see Figures S9 and S10. The density of states calculations reveal the introduction of localised states in the $\mathrm{TiO}_{2}$ band gap for the oxygen adsorbed systems. We identify those as antibonding $\pi^{*}$ states of $\mathrm{O}_{2}$. Importantly, the presence of intragap unoccupied $\mathrm{O}_{2}$ levels can lead to the formation of a charge-transfer (CT) complex with visible light or near infrared activation. This allows the direct transfer of electrons from the surface to the adsorbed $\mathrm{O}_{2}$ molecules following exposure to light. A visible-light activated CT complex based on nitrogen (II) oxide adsorbed on titania has been proposed and investigated before. ${ }^{36}$ Here, we investigate a CT process involving $\mathrm{O}_{2}$. The formation of the CT complex is further verified by calculating the absorption spectrum of the $\mathrm{O}_{2}-\mathrm{TiO}_{2}$ system using the linear response time-dependent density functional tight binding (TD-DFTB) method. ${ }^{37,38}$ 
The results are shown in Figure 2.

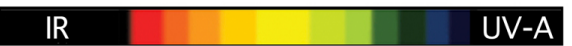

(a)

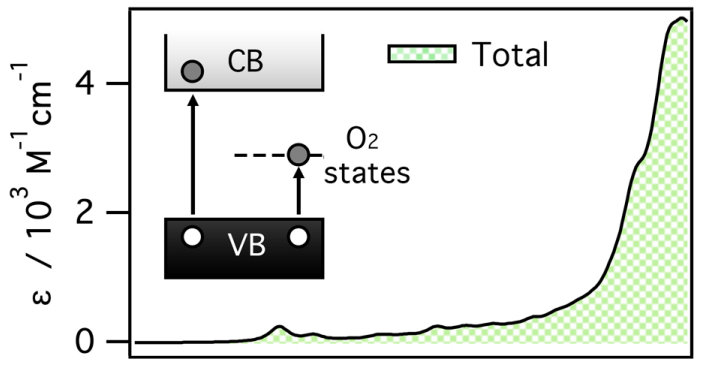

(b)

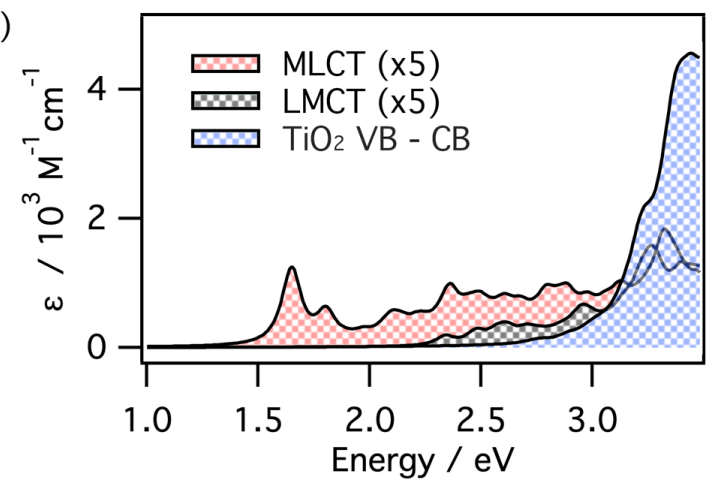

(c)
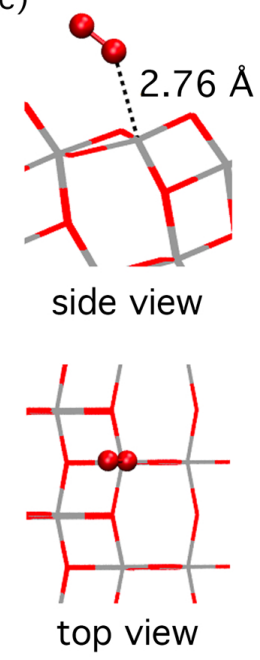

Figure 2: Absorption spectrum of the $\mathrm{O}_{2}-\mathrm{TiO}_{2}$ charge-transfer complex with a corresponding energy level diagram. The total absorption spectrum, (a), has been projected onto three separate components shown in (b); metal-to-ligand charge-transfer (MLCT) transitions (red), ligand-to-metal charge-transfer (LMCT) transitions (black) and band-to-band excitations (blue). $\epsilon$ corresponds to the molar extinction coefficient as discussed in reference. ${ }^{36}$ The adsorption geometry is shown to the right in (c) with oxygen and titanium atoms colored in red and silver respectively.

The spectrum was projected onto metal-to-ligand (ML) and ligand-to-metal (LM) CT excitations as well as transitions from the valence band to the conduction band, shown in Figure 2(b). We observe weak absorption bands in the visible region. Those correspond mainly to CT excitations from the top of the $\mathrm{TiO}_{2}$ valence band to the $\mathrm{O}_{2}$ molecular states, and to a lesser degree, transitions from the highest occupied molecular orbitals to the conduction band. The former is the dominant mechanism for the low energy part of the spectrum, and leads to formation of $\mathrm{O}_{2}^{-}$species and positive charge carriers at the surface. Figure S11 contains a plot directly comparing the absorption spectra of the pristine $\mathrm{TiO}_{2}$ and the $\mathrm{O}_{2}-\mathrm{TiO}_{2}$ systems, highlighting the formation of a new absorption peak at lower energies for 
the $\mathrm{O}_{2}-\mathrm{TiO}_{2}$ charge-transfer complex.

Oxygen adsorption on $\mathrm{TiO}_{2}$ can result in a number of species on the surface, including molecular $\mathrm{O}_{2}$, adsorbed $\mathrm{O}_{2}{ }^{-}, \mathrm{O}_{2}{ }^{2-}$ and, following dissociation, $\mathrm{O}^{2-}$. ${ }^{1}$ The latter three species may arise following the acceptance of excess electrons from the anatase surface, originating from intrinsic defects or subsurface dopants. ${ }^{1,39}$ The electron transfer between anatase $\mathrm{TiO}_{2}$ and $\mathrm{O}_{2}$ molecules after illumination with UV light was investigated using scanning probe microscopy (SPM). ${ }^{39}$ It was reported that when anatase(101) is exposed to molecular oxygen at $5.5 \mathrm{~K}$ two types of adsorbates appear; primarily neutral $\mathrm{O}_{2}$ molecules, which desorb at $64 \mathrm{~K}$, and activated $\mathrm{O}_{2}{ }^{-}$, which despite saturating at low coverages is significantly more stable and remains on the surface until above room temperature. An increase in the latter was observed following tip-induced electron injection and could also be created following photoinduced charge transfer from the substrate. It is known that physisorbed $\mathrm{O}_{2}$ is inert and must be excited to $\mathrm{O}_{2}{ }^{-}$via the acceptance of an electron before it can take part in chemical reactions. ${ }^{39}$

In the conventional picture, an explanation of how a $1.6 \mathrm{eV}$ photon laser can lead to the activation of oxygen and thus initiate the photocatalytic process is a non-linear twophoton absorption process leading to the generation of electron hole pairs in $\mathrm{TiO}_{2} \cdot{ }^{21,40}$ Such a process is to be expected under our experimental conditions and is in line with previous reports. Weak two (and three) photon absorption in bulk $\mathrm{TiO}_{2}$ has previously been reported for laser pulses with energies up to $3 \mu \mathrm{J}$ and wavelengths of $774 \mathrm{~nm}, 800 \mathrm{~nm}$ and $813 \mathrm{~nm}$ (pulse durations $290 \mathrm{fs}, 50 \mathrm{fs}$ and $174 \mathrm{fs}$ respectively) using a chirped-pulse amplified Ti:Sapphire system. ${ }^{40}$ In this scenario, electron hole pairs are generated in $\mathrm{TiO}_{2}$ upon light illumination following which they quickly localise at surface sites within 200 fs. ${ }^{4,41,42}$ Surface trapped electrons can then combine with surface trapped holes, between 1 and $10 \mathrm{ps},{ }^{3}$ or relax into deep bulk trapping sites with a 500 ps time constant, ${ }^{41}$ where they exhibit a long life time $(\mu \mathrm{s})$ but are unreactive. The shallow trapped species on the other hand are extremely reactive and are, in principle, available to interact with oxygen species on the surface. Holes can cause 
desorption of $\mathrm{O}_{2}{ }^{-}$species and the electrons can cause the charging of molecular oxygen species $\left(\right.$ to $\mathrm{O}_{2}{ }^{-}$) or alternatively cause dissociation of $\mathrm{O}_{2}{ }^{-}$. The physisorbed molecular state plays an important role in electron transfer. ${ }^{39}$ Studies have estimated that on bare $\mathrm{TiO}_{2}, \mathrm{O}_{2}$ from the gas phase captures electrons at times between 10 and $100 \mu$ s following light illumination, ${ }^{43}$ whilst surface trapped electrons in $\mathrm{TiO}_{2}$ in methanol solution interact with oxygen within 100 ns. ${ }^{44}$ This would have little chance to effectively compete with charge carrier recombination, or with charge carrier diffusion into subsurface traps. Our observation of photogenerated $\mathrm{CO}_{2}$ on the surface at 1.2 ps provides direct evidence that charge transfer to oxygen can occur on a much faster timescale than has been previously reported and our theoretical calculations provide evidence that an alternative mechanism, involving the formation of a charge-transfer complex and the direct transfer of electrons from $\mathrm{TiO}_{2}$ to $\mathrm{O}_{2}$, is the more realistic scenario under our experimental conditions at $1.6 \mathrm{eV}(770 \mathrm{~nm})$ photon energies. This newly proposed mechanism also explains how the photooxidation of CO can take place following a one-photon absorption process with photon energies below the band gap of the photocatalyst. Furthermore, the effective electron trapping by oxygen following the direct electron transfer hinders the recombination between the charge carriers. This is desirable for efficient photooxidation and represents an advantageous mechanism over the formation of short-lived e/h pairs at surface or bulk $\mathrm{TiO}_{2}$. The low probability of occurrence of the CT compared to band-to-band excitations at higher energies may be compensated by the effective electron trapping of the CT mechanism. This kind of compensation was shown for the degradation of $\mathrm{NO}$ on $\mathrm{TiO}_{2}$, for which photonic efficiencies, defined as the ratio of the reaction rate and the incident photon flux, under visible (CT process) and UV (e/h generation) irradiation have the same orders of magnitude. ${ }^{36}$

Analogous to our study, $\mathrm{CO}$ oxidation on $\mathrm{Ru}(0001)$ was investigated by ultrafast optical pump - soft X-ray probe absorption measurements. ${ }^{22,26}$ It was proposed that during the pump - probe cycle atomic oxygen is activated by a site change from a threefold hollow to a bridge site. New electronic states were observed in the X-ray absorption spectra with a 
transient increase on a time scale of $800 \pm 250$ fs, indicative of new adsorbed species and ascribed to a distribution of $\mathrm{OC}-\mathrm{O}$ bond lengths following the interaction of CO directly with atomic oxygen. ${ }^{22}$ Contrary to our system where a delay of $1.0 \pm 0.2$ ps is observed before the onset of $\mathrm{CO}_{2}$ formation, the increase in corresponding XAS signals appeared to begin directly at $0.00 \pm 0.25$ ps. Studies often highlight the importance of oxygen dissociation, claiming that the oxidation of $\mathrm{CO}$ occurs via the resultant oxygen adatoms. ${ }^{12,13}$ From our data, we can thus infer that the delay in the onset of $\mathrm{CO}_{2}$ formation corresponds to the time it takes for the adsorbed oxygen to become activated, via direct charge transfer from $\mathrm{TiO}_{2}$, and to then dissociate and create the necessary oxygen adatoms to react. The subsequent rise time of the $\mathrm{CO}_{2}$ signal of $600 \pm 200$ fs can then be assigned to the time it takes for the activated oxygen species, following photodissociation, to interact with a corresponding CO molecule. This is comparable to the $800 \pm 250$ fs transient reported for CO oxidation on the metallic Ru surface. ${ }^{22}$

\section{Conclusions}

In this study, we report on a photocatalytic surface reaction initiated by oxygen activation, monitored on an ultrafast timescale using femtosecond resolution X-ray photoemission spectroscopy and combined with theoretical calculations. The photooxidation of $\mathrm{CO}$ on the anatase $\mathrm{TiO}_{2}(101)$ surface leads to $\mathrm{CO}_{2}$ formation on the surface between 1.2 and $2.8( \pm 0.2)$ ps after light illumination. No long-lived intermediate species were observed leading up to this. Rather than the conventional photocatalytic process initiated by e/h pair generation at the $\mathrm{TiO}_{2}$ surface, our calculations suggest the existence of an alternative mechanism whereby the photoreaction can be initiated using a $1.6 \mathrm{eV}(770 \mathrm{~nm})$ photon laser following the formation of an $\mathrm{O}_{2}-\mathrm{TiO}_{2}$ charge-transfer complex. This allows the direct transfer of electrons from the top of the $\mathrm{TiO}_{2}$ valence band to $\mathrm{O}_{2}$ molecular states, leading to the activation of $\mathrm{O}_{2}$. These species are subsequently responsible for the photooxidation of adsorbed $\mathrm{CO}$ to $\mathrm{CO}_{2}$ 
within $1.2 \pm 0.2$ ps. Further to providing an optical induced channel for the activation of oxygen, and the subsequent photooxidation of $\mathrm{CO}$ to $\mathrm{CO}_{2}$, we propose that the formation of a charge-transfer complex also hinders charge carrier recombination. In addition to demonstrating the unique possibilities offered by soft X-ray free-electron lasers to directly follow and monitor photocatalytic reactions on oxide surfaces, this study acts as a crucial first step to improve the understanding of the early stage behaviour of photogenerated charge carriers and reaction intermediates in oxide photochemistry. This provides unique, and essential, real-time insight into the behaviour of the photogenerated charge carriers and the underlying mechanisms responsible for oxygen activation that has until now been unattainable. The activation of oxygen and the time-scale on which this occurs is important for a number of reactions that have important environmental implications for air purification, including the degradation of volatile organic compounds, viruses and pollutants. Acquisition of this knowledge is thus of paramount interest from both a fundamental scientific perspective and from an economical and environmental perspective.

\section{Experimental Method}

The experiment was performed at the second branch of the plane grating monochromator beamline (PG2) ${ }^{29,30}$ of the free-electron laser facility FLASH located at Deutsches Elektronen-

Synchrotron (DESY) in Hamburg, Germany. ${ }^{27,28}$ To measure core level XP spectra FLASH was operated with a fundamental wavelength of $5.74 \mathrm{~nm}(216 \mathrm{eV})$ and an average pulse energy of $30-40 \mu \mathrm{J}$. By tuning the monochromator the 3rd harmonic, radiation of $1.91 \mathrm{~nm}$ $(647.9 \mathrm{eV})$ could be used for the experiment. The FEL provided a pulse pattern of bursts with $10 \mathrm{~Hz}$ repetition rate. Each burst consist of 400 single X-ray pulses separated by 1 $\mu \mathrm{s}$ (a repetition rate of $1 \mathrm{MHz}$ ) giving a pulse rate of 4000 pulses/s on average. Each FEL pulse had a FWHM temporal width of $<100$ fs which experienced temporal stretching in the monochromator to approximately $150 \mathrm{fs}$. An optical laser of $770 \mathrm{~nm}(1.6 \mathrm{eV})$ providing the 
same pulse pattern as the FEL was used to pump the photocatalytic reaction. With a pulse

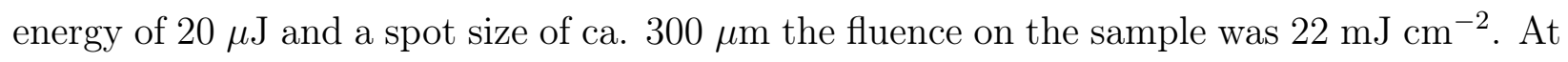
full fluence, the optical laser caused the immediate desorption of CO (see Figure S12) and damaged the sample surface, as evidenced by the formation of $\mathrm{Ti}^{3+}$ species. Correspondingly, the pump-laser was attenuated during the experiment to avoid this, resulting in a fluence of $4.4 \mathrm{~mJ} \mathrm{~cm}{ }^{-2}$. The pulses of the optical laser had a duration of $<100 \mathrm{fs}$ FWHM and were synchronized to the FEL pulses with a jitter in the range of $150 \mathrm{fs}$ FWHM. The timing offset of the optical pulses with respect to the FEL pulses was controlled by a mechanical delay stage.

The experimental (ultra-high vacuum) high efficient wide-angle electron spectrometer WESPE chamber was equipped with a heating stage and an ion gun for sample preparation, low energy electron diffraction (LEED) optics and a Themis 1000 high-resolution time of flight spectrometer equipped with a three-dimensional delay line detector (3D-DLD40404Q). The manipulator contained a cryostat, allowing the sample to be cooled with liquid He. There were three gas lines containing Ar (purity 99.999\%) for sample preparation and $\mathrm{CO}$ (purity $\geq 99.97 \%$ ) and $\mathrm{O}_{2}$ (purity 99.999\%) for the experiment. The anatase $\mathrm{TiO}_{2}(101)$ single crystal $\left(8 \mathrm{~mm} \times 8 \mathrm{~mm} \times 2 \mathrm{~mm}\right.$ ) was prepared by repeated $1 \mathrm{keV} \mathrm{Ar}{ }^{+}$ion bombardment and $850 \mathrm{~K}$ anneal cycles, in a backpressure of $1 \times 10^{-8}$ mbar $\mathrm{O}_{2}$, until X-ray photoelectron spectra showed the surface to be free of contamination (Figure S13) and a sharp (1x1) LEED pattern was obtained (Figure S14). For the final annealing step, the sample was left to cool in the back pressure of $\mathrm{O}_{2}$ before being exposed to a mixture of $\mathrm{CO} / \mathrm{O}_{2}\left(3 \times 10^{-8} \mathrm{mbar} / 3 \mathrm{x}\right.$ $10^{-8} \mathrm{mbar}$ ); this treatment produced the stoichiometric surface as evidenced by the absence of any $\mathrm{Ti}^{3+}$ species in the Ti $2 \mathrm{p}$ spectrum, which can usually be inferred from a low binding energy shoulder to the $\mathrm{Ti}^{4+}$ doublet. $^{5}$

All spectra were recorded at normal emission, at $60 \mathrm{~K}$ and with a $30 \mathrm{eV}$ pass energy. Since the sample was held at $60 \mathrm{~K}$, a significantly lower temperature than the surrounding chamber, the surface was subject to the unfortunate yet unavoidable adsorption of water 
over extended time periods as indicated by the growth of the peak at $533.4 \mathrm{eV}^{5}$ shown in Figure S8. The basis of the experiment relies on the fact that following photooxidation the $\mathrm{CO}_{2}$ desorbs from the surface and $\mathrm{CO}$ from the gas phase adsorbs. The presence of water is therefore problematic since it hinders CO adsorption, likely blocking adsorption sites. Long data acquisition times were required to obtain the necessary statistics and as such, to combat the saturation of the surface with water, the surface was flashed to $600 \mathrm{~K}$ every hour. This resulted in the removal of water and adventitious carbon, thus renewing the surface ${ }^{16}$ without inducing any modifications to the substrate (Figure S15). During data acquisition the incident pulses were rastered across the sample surface to limit laserinduced damage, doing so means that the observed fluctuation in $\mathrm{CO}$ concentration could be considered an artefact of a non-homogenous surface and the continuous probing of different sample positions. A consequence of this is that one can only interpret the data by comparing the concentrations of $\mathrm{CO}$ and $\mathrm{CO}_{2}$ and any change in absolute concentration cannot be considered a reliable source of information.

Taking into consideration the cross-section for photoionization at $647.9 \mathrm{eV},{ }^{35}$ the majority of the analysis has been carried out using the $\mathrm{O} 1$ s core levels owing to the improved statistics. Fitting of the XP spectra was carried out using CasaXPS, either with a shirley or a linear background in conjunction with Gaussian:Lorentzian(G:L) curves (0.7:0.3) to simulate the line shapes. To align the data on the binding energy scale the work function of the analyzer was calculated by aligning the Ti 2 p peak to $459.1 \mathrm{eV}$. This provided the work function, which was subsequently used to calculate the binding energies of the $\mathrm{C}$ 1s and $\mathrm{O}$ 1s core level peaks (with an associated error of $\pm 0.1 \mathrm{eV}$ ). The binding energies of $\mathrm{CO}$ and $\mathrm{CO}_{2}$ were determined prior to the experiment at FLASH using a lab based SPECS XPS instrument consisting of a monochromatic Al X-ray source $(\mathrm{h} \nu=1486.6 \mathrm{eV}$; anode operating at $14 \mathrm{kV})$ with a high-resolution two-dimensional delay line detector. ${ }^{16} \mathrm{XP}$ spectra were recorded in fixed transmission mode, with a pass energy of $30 \mathrm{eV}$, at normal emission and at a temperature of $98 \mathrm{~K}$. 


\section{Theoretical Calculations}

Density functional theory (DFT) periodic calculations in the PBE approach ${ }^{45}$ were performed according to the VASP code implementation. ${ }^{46-49}$ The anatase (101) surface was modelled with a $\mathrm{TiO}_{2}$ slab containing $36 \mathrm{Ti}$ atoms and $72 \mathrm{O}$ atoms in a supercell with 2x3 surface unit cells. The Brillouin zone was sampled with a 2x2x1 Monkhorst-Pack kpoints grid. We employed an energy cutoff of $400 \mathrm{eV}$ and the projected augmented-wave (PAW) method. ${ }^{50,51}$ In all calculations involving $\mathrm{O}_{2}$, the triplet spin configuration of this molecule was considered. Van der Waals interactions were accounted for by using the DFTD3 dispersion correction with Becke-Johnson damping. ${ }^{52,53}$ All minima were confirmed by frequency calculations. For the Density of States (DOS) analysis at the DFT level of theory a $(4 \times 4 \times 1)$ k-points grid was used. A PBE functional ${ }^{45}$ together with semi-empirical nonlocal external potentials (NLEP) ${ }^{54-56}$ was employed. For the DOS analysis using the DFTB $\operatorname{approach}^{57}$ a $(4 \times 4 \times 1)$ k-points grid was used. The reported adsorption energy is defined as $E_{a d s}=E_{\text {comp }}-E_{\text {surf }}-E_{\text {mol }}$, where $E_{\text {surf }}, E_{\text {mol }}$ and $E_{\text {comp }}$ are, respectively, the total energies of the $\mathrm{TiO}_{2}$ surface, the isolated molecule(s) and the compound system formed by the adsorbates and the substrate. Within this definition, a negative value of the adsorption energy indicates an exothermic process.

For the computation of the absorption spectra at the TD-DFTB level, we employed the $\mathrm{DFTB}+\operatorname{code}^{57}$ and the tiorg-0 -1 set of parameters. ${ }^{58}$ The repulsive potential for the Ti-O pair has been improved here for a better description of the physisorption of $\mathrm{O}_{2}$ on $\mathrm{TiO}_{2}$. The anatase(101) surface was modelled using a $\mathrm{Ti}_{24} \mathrm{O}_{71} \mathrm{H}_{46}$ cluster. The peripheral $\mathrm{O}$ atoms that do not belong to the (101) surface were saturated with H atoms to keep charge neutrality and to passivate dangling bonds states. The employed cluster model has been validated with periodic boundary condition calculations in terms of binding geometries and electronic structures. In the absorption spectra, the first 700 excitation energies of the $\mathrm{TiO}_{2}-\mathrm{O}_{2}$ system were computed. This covers an energetic region of up to $3.7 \mathrm{eV}$. We considered only excited states with a spin contamination of the expectation value of the spin square operator, $<S^{2}>$, 
lower than 0.5 .

\section{Acknowledgement}

This paper is dedicated to our dear colleague Prof. Wilfried Wurth, leading scientist of the free-electron laser FLASH and lead scientist at DESY, who passed away unexpectedly on May 8, 2019. Wilfried Wurth pioneered time resolved photoemission at FLASH, which is the basis for the experimental part of this study. We appreciate his outstanding contribution to the field and miss the extremely valuable scientific discussions with him.

MW acknowledges support from the Initiative and Networking Funds of the Helmholtz Association through ExNet-0002. This work is supported by the DFG within the excellence cluster Center for Ultrafast Imaging (CUI). AD and AR acknowledge financial support from the European Union's Horizon 2020 research and innovation program under Marie Sklodowska-Curie grant agreement no. 753874 and from the European Research Council(ERC-2015-AdG-694097), Grupos Consolidados (IT1249-19), and SFB925. TF and AD acknowledge financial support from NSAF U1930402 and computational resources from the Beijing Computational Science Research Center. Further, we acknowledge the excellent support from the scientific and technical staff of FLASH and thank Dr. Martin Beye for his useful discussions and comments.

\section{Supporting Information Available}

The following files are available free of charge.

- S1: XP spectra of the C 1s and O 1s core levels showing the adsorption of CO (red) and $\mathrm{CO}_{2}$ (blue) on anatase $\mathrm{TiO}_{2}(101)(\mathrm{h} \nu=1486.6 \mathrm{eV}, \mathrm{T}=98 \mathrm{~K}$, normal emission)

- S2: XP spectra of the C 1s core level showing the adsorption and subsequent oxidation of $\mathrm{CO}$ (red) to $\mathrm{CO}_{2}$ (blue) on anatase $\mathrm{TiO}_{2}(101)(\mathrm{h} \nu=1486.6 \mathrm{eV}, \mathrm{T}=98 \mathrm{~K}$, normal 
emission)

- S3: XP spectra of the C 1s core level showing the effect of oxygen and X-ray exposure, in the absence of UV light, for $\mathrm{CO}$ on anatase $\mathrm{TiO}_{2}(101)(\mathrm{h} \nu=1486.6 \mathrm{eV}, \mathrm{T}=98 \mathrm{~K}$, normal emission).

- S4: (a) time resolved XP map of the Ti $2 \mathrm{p}_{3 / 2}$ core level $\left(\mathrm{h} \nu_{F E L}=647.9 \mathrm{eV}, \mathrm{h} \nu_{\text {opt }}=\right.$ $1.6 \mathrm{eV}, \mathrm{T}=60 \mathrm{~K})$ as a function of delay time. The counts over the peak maxima were integrated and plotted against delay time in (b). (c) shows the Ti $2 \mathrm{p}$ spectra extracted in a 200 fs window corresponding to a time off time zero (bottom) and on time zero (top).

- S5: Time resolved XP spectrum of the O 1s core level $\left(\mathrm{h} \nu_{F E L}=647.9 \mathrm{eV}, \mathrm{h} \nu_{\text {opt }}=1.6\right.$ $\mathrm{eV}, \mathrm{T}=60 \mathrm{~K}$ ) extracted on time zero in a $200 \mathrm{fs}$ window.

- S6: Time resolved XP spectra of the O 1s core level during the photooxidation of CO to $\mathrm{CO}_{2}$ on $\mathrm{TiO}_{2}(101)\left(\mathrm{h} \nu_{F E L}=647.9 \mathrm{eV}, \mathrm{h} \nu_{\text {opt }}=1.6 \mathrm{eV}, \mathrm{T}=60 \mathrm{~K}\right)$ displayed over a broader binding energy range.

- S7: Time resolved XP spectra of the O 1s core level during the photooxidation of CO to $\mathrm{CO}_{2}$ on $\mathrm{TiO}_{2}(101)\left(\mathrm{h} \nu_{F E L}=647.9 \mathrm{eV}, \mathrm{h} \nu_{\text {opt }}=1.6 \mathrm{eV}, \mathrm{T}=60 \mathrm{~K}\right)$ integrated over a 1 ps window.

- S8: time averaged XP spectra showing the adsorption of water, at $533.5 \mathrm{eV}$, on the surface over a 2 hour time period $\left(\mathrm{h} \nu_{F E L}=647.9 \mathrm{eV}, \mathrm{h} \nu_{o p t}=1.6 \mathrm{eV}, \mathrm{T}=60 \mathrm{~K}\right)$.

- S9: DFT calculations describing the electronic and structural properties of the adsorption of $\mathrm{CO}$ and $\mathrm{O}_{2}$

- S10: (a) The theoretically calculated density of states for $\mathrm{O}_{2}$ adsorption, $\mathrm{O}_{2} / \mathrm{CO}$ coadsorption and for the pristine $\mathrm{TiO}_{2}$ surface using DFTB approach. (b) The density of 
states for the $\mathrm{O}_{2}$ adsorbed system using the Perdew-Burke-Ernzerhof functional at the DFT level of theory.

- S11: Absorption spectra comparing the pristine $\mathrm{TiO}_{2}$ and the $\mathrm{O}_{2}-\mathrm{TiO}_{2}$ systems.

- S12: Time averaged XP spectra of the O 1s core level showing the laser induced desorption of $\mathrm{CO}\left(\mathrm{h} \nu_{F E L}=647.9 \mathrm{eV}, \mathrm{h} \nu_{\text {opt }}=1.6 \mathrm{eV}, \mathrm{T}=60 \mathrm{~K}\right)$.

- S13: XP spectra of the Ti 2p, O 1s and C 1s core levels spectra for the as prepared anatase $\mathrm{TiO}_{2}(101)$ surface $\left(\mathrm{h} \nu_{F E L}=647.9 \mathrm{eV}, \mathrm{T}=60 \mathrm{~K}\right)$.

- S14: (1x1) LEED pattern for the as prepared anatase $\mathrm{TiO}_{2}(101)$ surface

- S15: (a) XP spectra of the Ti $2 \mathrm{p}$ core level for the as prepared anatase $\mathrm{TiO}_{2}(101)$ surface before (bottom) and after (top) flashing the surface to $600 \mathrm{~K}$ ( $\mathrm{h} \nu=1486.6 \mathrm{eV}$, $\mathrm{T}=98 \mathrm{~K}$, normal emission). (b) corresponding difference spectrum.

\section{References}

(1) Li, Y.-F.; Aschauer, U.; Chen, J.; Selloni, A. Adsorption and reactions of $\mathrm{O}_{2}$ on anatase $\mathrm{TiO}_{2}$. Acc. Chem. Res. 2014, 47, 3361-3368.

(2) Linsebigler, A. L.; Lu, G.; Yates, J. T. Photocatalysis on $\mathrm{TiO}_{2}$ surfaces: principles, mechanisms, and selected results. Chem. Rev. 1995, 95, 735-758.

(3) Schneider, J.; Matsuoka, M.; Takeuchi, M.; Zhang, J.; Horiuchi, Y.; Anpo, M.; Bahnemann, D. W. Understanding $\mathrm{TiO}_{2}$ photocatalysis: mechanisms and materials. Chem. Rev. 2014, 114, 9919-9986.

(4) Kohtani, S.; Kawashima, A.; Miyabe, H. Reactivity of trapped and accumulated electrons in titanium dioxide photocatalysis. Catalysts 2017, 7, 303.

(5) Diebold, U. The surface science of titanium dioxide. Surf. Sci. Rep. 2003, 48, 53-229. 
(6) Fujishima, A.; Zhang, X.; Tryk, D. A. $\mathrm{TiO}_{2}$ photocatalysis and related surface phenomena. Surf. Sci. Rep. 2008, 63, 515-582.

(7) Fuhishima, A.; Honda, K. Electrochemical photolysis of water at a semiconductor electrode. Nature 1972, 238, 37-38.

(8) Setvin, M.; Aschauer, U.; Hulva, J.; Simschitz, T.; Daniel, B.; Schmid, M.; Selloni, A.; Diebold, U. Following the reduction of oxygen on $\mathrm{TiO}_{2}$ anatase (101) step by step. J. Am. Chem. Soc. 2016, 138, 9565-9571.

(9) Linsebigler, A.; Lu, G.; Yates, J. T. CO Photooxidation on $\mathrm{TiO}_{2}(110)$. J. Phys. Chem. 1996, 100, 6631-6636.

(10) Xu, M.; Gao, Y.; Moreno, E. M.; Kunst, M.; Muhler, M.; Wang, Y.; Idriss, H.; Wöll, C. Photocatalytic activity of bulk $\mathrm{TiO}_{2}$ anatase and rutile single crystals using infrared absorption spectroscopy. Phys. Rev. Lett. 2011, 106, 138302-.

(11) Ovcharov, M. L.; Granchak, V. M. Photocatalytic activation of carbon monoxide on semiconductors and derived nanocomposites: basic principles and mechanisms: a review. Theor. Exp. Chem. 2019, 55, 173-200.

(12) Mitsuhara, K.; Tagami, M.; Matsuda, T.; Visikovskiy, A.; Takizawa, M.; Kido, Y. The mechanism of emerging catalytic activity of gold nano-clusters on rutile $\mathrm{TiO}_{2}(110)$ in CO oxidation reaction. J. Chem. Phys. 2012, 136, 124303.

(13) Yu, Y.-Y.; Gong, X.-Q. CO Oxidation at rutile $\mathrm{TiO}_{2}(110)$ : role of oxygen vacancies and titanium interstitials. ACS Catal. 2015, 5, 2042-2050.

(14) Czupryn, K.; Kocemba, I.; Rynkowski, J. Photocatalytic CO oxidation with water over Pt/ $\mathrm{TiO}_{2}$ catalysts. Reac. Kinet. Mech. Cat. 2018, 124, 187-201.

(15) Omaye, S. T. Metabolic modulation of carbon monoxide toxicity. Toxicology 2002, 180, $139-150$. 
(16) Wagstaffe, M.; Noei, H.; Stierle, A. Elucidating the defect-induced changes in the photocatalytic activity of $\mathrm{TiO}_{2}$. J. Phys. Chem. C 2020, 124, 12539-12547.

(17) Xu, M.; Noei, H.; Fink, K.; Muhler, M.; Wang, Y.; Wöll, C. The surface science approach for understanding reactions on oxide powders: the importance of IR spectroscopy. Angew. Chem. Int. Ed. 2012, 51, 4731-4734.

(18) Rohmann, C.; Wang, Y.; Muhler, M.; Metson, J.; Idriss, H.; Wöll, C. Direct monitoring of photo-induced reactions on well-defined metal oxide surfaces using vibrational spectroscopy. Chem. Phys. Lett. 2008, 460, 10-12.

(19) Zhao, H.; Zhang, Q.; Weng, Y.-X. Deep surface trap filling by photoinduced carriers and interparticle electron transport observed in $\mathrm{TiO}_{2}$ nanocrystalline film with timeresolved visible and mid-IR transient spectroscopies. J. Phys. Chem. C 2007, 111, $3762-3769$.

(20) Yamakata, A.; Ishibashi, T.-a.; Onishi, H. Electron- and hole-capture reactions on $\mathrm{Pt} / \mathrm{TiO}_{2}$ photocatalyst exposed to methanol vapor studied with time-resolved infrared absorption spectroscopy. J. Phys. Chem. B 2002, 106, 9122-9125.

(21) Ozawa, K.; Emori, M.; Yamamoto, S.; Yukawa, R.; Yamamoto, S.; Hobara, R.; Fujikawa, K.; Sakama, H.; Matsuda, I. Electron-hole recombination time at $\mathrm{TiO}_{2}$ singlecrystal surfaces: influence of surface band bending. J. Phys. Chem. Lett. 2014, 5, $1953-1957$.

(22) Öström, H.; Öberg, H.; Xin, H.; LaRue, J.; Beye, M.; Dell'Angela, M.; Gladh, J.; Ng, M. L.; Sellberg, J. A.; Kaya, S. et al. Probing the transition state region in catalytic CO oxidation on Ru. Science 2015, 347, 978.

(23) Beye, M.; Anniyev, T.; Coffee, R.; Dell'Angela, M.; Föhlisch, A.; Gladh, J.; Katayama, T.; Kaya, S.; Krupin, O.; Møgelhøj, A. et al. Selective ultrafast probing 
of transient hot chemisorbed and precursor states of $\mathrm{CO}$ on $\mathrm{Ru}(0001)$. Phys. Rev. Lett. 2013, 110, 186101-.

(24) Mancuso, A. P.; Gorniak, T.; Staier, F.; Yefanov, O. M.; Barth, R.; Christophis, C.; Reime, B.; Gulden, J.; Singer, A.; Pettit, M. E. et al. Coherent imaging of biological samples with femtosecond pulses at the free-electron laser FLASH. New J. Phys. 2010, 12, 035003-035003.

(25) Gahl, C.; Azima, A.; Beye, M.; Deppe, M.; Döbrich, K.; Hasslinger, U.; Hennies, F.; Melnikov, A.; Nagasono, M.; Pietzsch, A. et al. A femtosecond X-ray/optical crosscorrelator. Nat. Photonics 2008, 2, $165 \mathrm{EP}-$.

(26) Dell'Angela, M.; Anniyev, T.; Beye, M.; Coffee, R.; Föhlisch, A.; Gladh, J.; Katayama, T.; Kaya, S.; Krupin, O.; LaRue, J. et al. Real-time observation of surface bond breaking with an X-ray laser. Science 2013, 339, 1302.

(27) Toleikis, S. The FLASH facility current status in 2018 and future upgrade plans. AIP Conference Proceedings 2019, $2054,030015$.

(28) Ackermann, W.; Asova, G.; Ayvazyan, V.; Azima, A.; Baboi, N.; Bähr, J.; Balandin, V.; Beutner, B.; Brandt, A.; Bolzmann, A. et al. Operation of a free-electron laser from the extreme ultraviolet to the water window. Nat. Photonics 2007, 1, 336-342.

(29) Martins, M.; Wellhöfer, M.; Hoeft, J. T.; Wurth, W.; Feldhaus, J.; Follath, R. Monochromator beamline for FLASH. Rev. Sci. Instrum. 2006, r7, 115108.

(30) Gerasimova, N.; Dziarzhytski, S.; Feldhaus, J. The monochromator beamline at FLASH: performance, capabilities and upgrade plans. J. Mod. Opt. 2011, 58, 14801485.

(31) Pietzsch, A.; Föhlisch, A.; Beye, M.; Deppe, M.; Hennies, F.; Nagasono, M.; Suljoti, E.; Wurth, W.; Gahl, C.; Döbrich, K. et al. Towards time resolved core level photoelec- 
tron spectroscopy with femtosecond X-ray free-electron lasers. New J. Phys. 2008, 10, 033004-033004.

(32) Setvin, M.; Buchholz, M.; Hou, W.; Zhang, C.; Stöger, B.; Hulva, J.; Simschitz, T.; Shi, X.; Pavelec, J.; Parkinson, G. S. et al. A multitechnique study of CO adsorption on the $\mathrm{TiO}_{2}$ anatase (101) surface. J. Phys. Chem. C 2015, 119, 21044-21052.

(33) Yu, Y.; Mao, B.; Geller, A.; Chang, R.; Gaskell, K.; Liu, Z.; Eichhorn, B. W. CO activation and carbonate intermediates: an operando AP-XPS study of $\mathrm{CO}_{2}$ electrolysis reactions on solid oxide electrochemical cells. Phys. Chem. Chem. Phys. 2014, 16, $11633-11639$.

(34) Briggs, D.; Beamson, G. XPS studies of the oxygen 1s and 2 s levels in a wide range of functional polymers. Anal. Chem. 1993, 65, 1517-1523.

(35) McMaster, W. H.; Del Grande, N. K.; Mallett, J. H.; Hubbell, J. H. Compilation of X-ray cross sections; 1969.

(36) Freitag, J.; Domínguez, A.; Niehaus, T. A.; Hülsewig, A.; Dillert, R.; Frauenheim, T.; Bahnemann, D. W. Nitrogen(II) oxide charge transfer complexes on $\mathrm{TiO}_{2}$ : a new source for visible-light activity. J. Phys. Chem. C 2015, 119, 4488-4501.

(37) Niehaus, T. A.; Suhai, S.; Della Sala, F.; Lugli, P.; Elstner, M.; Seifert, G.; Frauenheim, T. Tight-binding approach to time-dependent density-functional response theory. Phys. Rev. B 2001, 63, 085108-.

(38) Domínguez, A.; Aradi, B.; Frauenheim, T.; Lutsker, V.; Niehaus, T. A. Extensions of the time-dependent density functional based tight-binding approach. J. Chem. Theory Comput. 2013, 9, 4901-4914.

(39) Setvin, M.; Hulva, J.; Parkinson, G. S.; Schmid, M.; Diebold, U. Electron transfer 
between anatase $\mathrm{TiO}_{2}$ and an $\mathrm{O}_{2}$ molecule directly observed by atomic force microscopy. Proc. Natl. Acad. Sci. U.S.A. 2017, 114, E2556.

(40) Evans, C. C.; Bradley, J. D. B.; Martí-Panameño, E. A.; Mazur, E. Mixed two- and three-photon absorption in bulk rutile $\left(\mathrm{TiO}_{2}\right)$ around $800 \mathrm{~nm}$. Optics Express $\mathbf{2 0 1 2}$, 20, 3118-3128.

(41) Tamaki, Y.; Furube, A.; Murai, M.; Hara, K.; Katoh, R.; Tachiya, M. Dynamics of efficient electron-hole separation in $\mathrm{TiO}_{2}$ nanoparticles revealed by femtosecond transient absorption spectroscopy under the weak-excitation condition. Phys. Chem. Chem. Phys. 2007, 9, 1453-1460.

(42) Yang, X.; Tamai, N. How fast is interfacial hole transfer? In situ monitoring of carrier dynamics in anatase $\mathrm{TiO}_{2}$ nanoparticles by femtosecond laser spectroscopy. Phys. Chem. Chem. Phys. 2001, 3, 3393-3398.

(43) Yamakata, A.; Ishibashi, T.-a.; Onishi, H. Water- and oxygen-induced decay kinetics of photogenerated electrons in $\mathrm{TiO}_{2}$ and $\mathrm{Pt} / \mathrm{TiO}_{2}$ : a time-resolved infrared absorption study. J. Phys. Chem. B 2001, 105, 7258-7262.

(44) Yoshihara, T.; Katoh, R.; Furube, A.; Tamaki, Y.; Murai, M.; Hara, K.; Murata, S.; Arakawa, H.; Tachiya, M. Identification of reactive species in photoexcited nanocrystalline $\mathrm{TiO}_{2}$ films by wide-wavelength-range (400-2500 nm) transient absorption spectroscopy. J. Phys. Chem. B 2004, 108, 3817-3823.

(45) Perdew, J. P.; Ernzerhof, M.; Burke, K. Rationale for mixing exact exchange with density functional approximations. J. Chem. Phys. 1996, 105, 9982-9985.

(46) Kresse, G.; Hafner, J. Ab initio molecular dynamics for liquid metals. Phys. Rev. B 1993, 47, 558-561. 
(47) Kresse, G.; Hafner, J. Ab initio molecular-dynamics simulation of the liquid-metalamorphous-semiconductor transition in germanium. Phys. Rev. B 1994, 49, 1425114269.

(48) Kresse, G.; Furthmüller, J. Efficiency of ab-initio total energy calculations for metals and semiconductors using a plane-wave basis set. Comput. Mater. Sci. 1996, 6, 15-50.

(49) Kresse, G.; Furthmüller, J. Efficient iterative schemes for ab initio total-energy calculations using a plane-wave basis set. Phys. Rev. B 1996, 54, 11169-11186.

(50) Blöchl, P. E. Projector augmented-wave method. Phys. Rev. B 1994, 50, 17953-17979.

(51) Kresse, G.; Joubert, D. From ultrasoft pseudopotentials to the projector augmentedwave method. Phys. Rev. B 1999, 59, 1758-1775.

(52) Grimme, S.; Antony, J.; Ehrlich, S.; Krieg, H. A consistent and accurate ab initio parametrization of density functional dispersion correction (DFT-D) for the 94 elements H-Pu. The Journal of Chemical Physics 2010, 132, 154104.

(53) Grimme, S.; Ehrlich, S.; Goerigk, L. Effect of the damping function in dispersion corrected density functional theory. Journal of Computational Chemistry 2011, 32, 14561465.

(54) Farzalipour Tabriz, M.; Aradi, B.; Frauenheim, T.; Deák, P. Application of the LanyZunger polaron correction for calculating surface charge trapping. J. Phys.: Condens. Matter 2017, 29, 394001.

(55) Lany, S.; Raebiger, H.; Zunger, A. Magnetic interactions of Cr-Cr and Co-Co impurity pairs in $\mathrm{ZnO}$ within a band-gap corrected density functional approach. Physical Review B 2008, r77, 241201-.

(56) Lany, S. Semiconducting transition metal oxides. J. Phys.: Condens. Matter 2015, 27, 283203. 
(57) Aradi, B.; Hourahine, B.; Frauenheim, T. DFTB+, a Sparse matrix-based implementation of the DFTB method. J. Phys. Chem. A 2007, 111, 5678-5684.

(58) Dolgonos, G.; Aradi, B.; Moreira, N. H.; Frauenheim, T. An improved self-consistentcharge density-functional tight-binding (SCC-DFTB) set of parameters for simulation of bulk and molecular systems involving titanium. J. Chem. Theory Comput. 2010, 6, $266-278$. 
Graphical TOC Entry

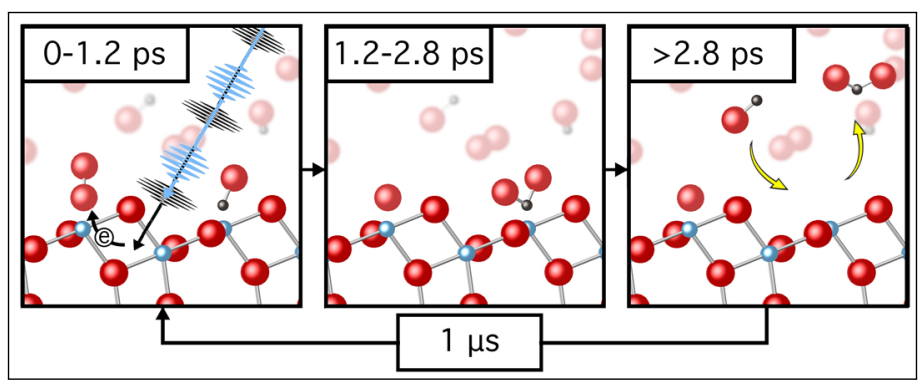

